\title{
Multi-threshold second-order phase transition in laser
}

\author{
ZHUANG Wei ${ }^{1}$, YU DeShui ${ }^{1}$, LIU ZhiWen $^{2} \&$ CHEN JingBiao $^{1^{*}}$ \\ ${ }^{1}$ Institute of Quantum Electronics, and State Key Laboratory of Advanced Optical Communication System \& Network, School of Electronics \\ Engineering \& Computer Science, Peking University, Beijing 100871, China; \\ ${ }^{2}$ Department of Electrical Engineering, The Pennsylvania State University, University Park, PA 16802, USA
}

Received June 23, 2011; accepted August 4, 2011

\begin{abstract}
We present a theory of the multi-threshold second-order phase transition, and experimentally demonstrate the multi-threshold secondorder phase transition phenomenon. With carefully selected parameters, in an external cavity diode laser system, we observe secondorder phase transition with multiple (three or four) thresholds in the measured power-current-temperature three dimensional phase diagram. Such controlled death and revival of second-order phase transition sheds new insight into the nature of ubiquitous secondorder phase transition. Our theory and experiment show that the single threshold second-order phase transition is only a special case of the more general multi-threshold second-order phase transition, which is an even richer phenomenon.
\end{abstract}

second-order phase transition, external cavity diode laser, Ginzburg-Landau free energy

Citation: Zhuang W, Yu D S, Liu Z W, et al. Multi-threshold second-order phase transition in laser. Chinese Sci Bull, 2011, 56: 3812-3816, doi: 10.1007/s11434-0114826-Z

Phase transition is an important interdisciplinary research field, which includes the phase transitions of liquid-vapor [1], networks [2], polymer chain [3], circuit QED [4], superconductivity [5,6] and laser. Particularly, Landau's theory [5,6] of the second order phase transition (SOPT) is crucial for a variety of important physical systems, including helium superfluidity [7-9], superconductivity [10], ferromagnetic system $[11,12]$, Bose-Einstein condensates [13], as well as lasers [14-20]. However, all of these SOPTs observed in experiments or investigated in theories so far possess only a single critical point (or threshold). In other words, there is only one critical point for symmetry breaking [5].

In this work, we present a theory of the multi-threshold SOPT, and demonstrate experimentally the multi-threshold SOPT phenomenon in an external cavity diode laser system with carefully selected parameters. Our results show that the single threshold SOPT is only a special case of the more general multi-threshold SOPT. Our theory and experiment imply that previous studied fascinating SOPTs, such as spontaneous magnetization [21], superfluidity [22,23] and superconductivity [24-26], which are solely limited to single critical-point

*Corresponding author (email: jbchen@pku.edu.cn) behavior, may be extended to the multi-threshold SOPT.

\section{Theory}

The Landau expression for the free energy $F$ near the critical point $[5,6]$, a function of an order parameter $\psi$,

$$
F=F_{n}+\alpha|\psi|^{2}+\frac{\beta}{2}|\psi|^{4}
$$

is generally applied to all aforementioned SOPTs. Here, the coefficient $\alpha=\alpha_{c}\left(T-T_{c}\right)$, is assumed by Landau to be a simple linear function of temperature $T$ when $T$ is lower than the critical temperature $T_{c} . F_{n}$ is the free energy of the normal phase.

However, if the coefficient $\alpha$ consists of periodic function of $T$, for example, a trigonometric function, the system can evolve to a multi-threshold SOPT phenomenon with suitable experimental parameters, as demonstrated in this paper. In this case, the free energy is expressed as

$$
F=F_{n}+\alpha_{c}\left(T-T_{c}-T_{m c} \cos \phi(T)\right)|\psi|^{2}+\frac{\beta}{2}|\psi|^{4}
$$


Each period of the cosine function of $T$ produces a threshold. As we will see later, in this case the order parameter $\psi$ will be a function with a periodic pattern of $T$. If $\phi(T)$ only weakly depends on the temperature $T$, eq. (2) is reduced to eq. (1).

The lasing action of a laser can be considered as a BoseEinstein condensation of photons, which also belongs to the SOPT. The analogies with superconductivity and ferromagnet, developed by Haken, Scully and others [14-20], show that the effective Ginzburg-Landau free energy of laser is

$$
G=G_{0}-\frac{1}{4}(A-C)|u|^{2}+\frac{1}{8} B|u|^{4}=G_{0}-\frac{1}{4}(A-C) n+\frac{1}{8} B|n|^{2},
$$

where the amplitude of electric field $u$ corresponds to the order parameter $\psi$ in eqs. (1) and (2), $G_{0}$ corresponds to $F_{n}$. $A$ is the gain in the active medium, $B$ is the saturation coefficient, $C$ is the cavity loss rate, and the photon density in laser cavity is $n$.

In order to experimentally demonstrate the multi-threshold SOPT in a laser, the key challenge is to carefully set the gain and cavity loss term $A-C$ in eq. (3) to be a periodic function of the effective temperature (the population inversion in laser corresponds to negative temperature [27]), which is related to injection current I in a diode laser.

Since the refractive index of semiconductor chip depends on the injection current, it can be shown that for a diode laser system,

$$
A-C=\alpha_{c}\left(I-I_{c}-I_{m c} \cos \phi(I)\right) .
$$

In Landau's theory, the dependence of the order parameter on the effective temperature near the critical point is determined by the condition that the free energy as a function of the order parameter is minimized $[5,6,10]$. For a laser near the threshold, from eq. (3) we obtain

$$
n=\frac{A-C}{B} \text {. }
$$

Near and above threshold, the exact solution of the photon density obtained from general laser rate equations involving carrier density $N$ and photon density $n$ in a diode laser,

$$
\begin{gathered}
\frac{\mathrm{d} N}{\mathrm{~d} t}=\frac{I}{q V}-\frac{N}{\tau_{N}}-g_{N}\left(N-N_{t r}\right) n, \\
\frac{\mathrm{d} n}{\mathrm{~d} t}=\Gamma g_{N}\left(N-N_{t r}\right) n-\frac{n}{\tau_{p}},
\end{gathered}
$$

should be

$$
n=\frac{A}{C} \frac{A-C}{B} .
$$

Here the gain $A$ and saturation coefficient $B$ are

$$
\begin{gathered}
A=\frac{\Gamma g_{N} \tau_{N}}{q V} I-\Gamma g_{N} N_{t r}, \\
B=\frac{\Gamma\left(g_{N} \tau_{N}\right)^{2}}{q V} I-\Gamma\left(g_{N}\right)^{2} \tau_{N} N_{t r} .
\end{gathered}
$$

Particularly, the cavity loss rate $C$ is a trigonometric function of $I$ and is given by

$$
C=C_{1}-C_{2} \cos \phi(I)
$$

$$
\phi(I)=\phi_{0}+\frac{\alpha_{H} l_{d}}{v_{g} \Gamma} C+\frac{2 \omega l_{d}}{c} \frac{\partial \eta}{\partial I} I,
$$

where $C_{1}=v_{g} \alpha_{i}+\frac{v_{g}}{l_{d}} \ln R_{c}, C_{2}=\frac{v_{g}}{l_{d}} \frac{R_{m}}{R_{c}}, R_{c}=\sqrt{\frac{1-R_{2} R_{3}}{R_{1}\left(R_{3}-R_{2}\right)}}$, $R_{m}=\sqrt{\frac{R_{2}}{R_{1}}} \frac{1-R_{3}}{R_{3}-R_{2}}, q$ is the electron charge, $\phi_{0}$ is a constant phase, and other symbols are listed in Table 1. Eq. (13) describes the effect of injection current through thermal effect and threshold gain through carrier density on the refractive index [28, 29].

\section{Experiments}

It should be noted that, eq. (8) has the same form as the exact solution of laser quantum theory [20]. This expression of eq. (8) can be reduced to eq. (5) when laser is near threshold, i.e. $A \approx C$. The measured output power of laser is hence given by $P=\kappa C n h v V_{p}$, where $\kappa$ is the output coupling efficiency, $h v$ is the photon energy, $V_{p}$ is the light field mode volume.

In an external cavity diode laser (ECDL) system, with carefully selected parameters for realizing a desired $\phi(I)$ in $A-C$ expression, we observed SOPT with multiple (three or four) thresholds in the measured power-current $(P-I)$ relationship, which corresponds to $\psi-T$ relation derived from eq. (1) in Landau's theory. The gradual evolvement from the SOPT with multiple critical points to single critical point SOPT confirmed experimentally and agrees well with eqs. (3) and (4). Figure 1(a) shows the measured three-dimensional (3D) phase diagrams of multi-threshold SOPT of the ECDL's output power as a function of the diode temperature $T_{d}$ and the injection current $I$. It can be clearly seen that up to four lasing

Table 1 Typical parameters of semiconductor diode [28, 29] used in calculations

\begin{tabular}{cll}
\hline Parameter & Description & Value \\
\hline$\Gamma$ & Optical confinement factor & 0.44 \\
$g_{N}$ & Gain coefficient & $3 \times 10^{-12} \mathrm{~m}^{3} / \mathrm{s}$ \\
$\tau_{N}$ & Carrier lifetime & $3 \mathrm{~ns}$ \\
$l_{d}$ & Active region length & $3 \times 10^{-4} \mathrm{~m}$ \\
$V$ & Active region volume & $6 \times 10^{-17} \mathrm{~m}^{3}$ \\
$N_{t r}$ & Transparency carrier density & $2 \times 10^{24} \mathrm{~m}^{-3}$ \\
$v_{g}$ & Group light speed & $7 \times 10^{7} \mathrm{~m} / \mathrm{s}$ \\
$\alpha_{i}$ & Internal material loss & $1000 \mathrm{~m}^{-1}$ \\
$\eta_{0}$ & Refractive index & 3.5 \\
$\alpha_{H}$ & Linewidth enhancement factor & 6 \\
$R_{1}$ & Facet reflectivity & 0.3 \\
$R_{2}$ & AR coated facet reflectivity & $5 \times 10^{-3}$ \\
$R_{3}$ & External cavity reflectivity & 0.05 \\
$\frac{\partial \eta}{\partial I}$ & Refractive index coefficient & 0.1 \\
$\lambda$ & Output wavelength & $830 \mathrm{~nm}^{2}$ \\
\hline
\end{tabular}



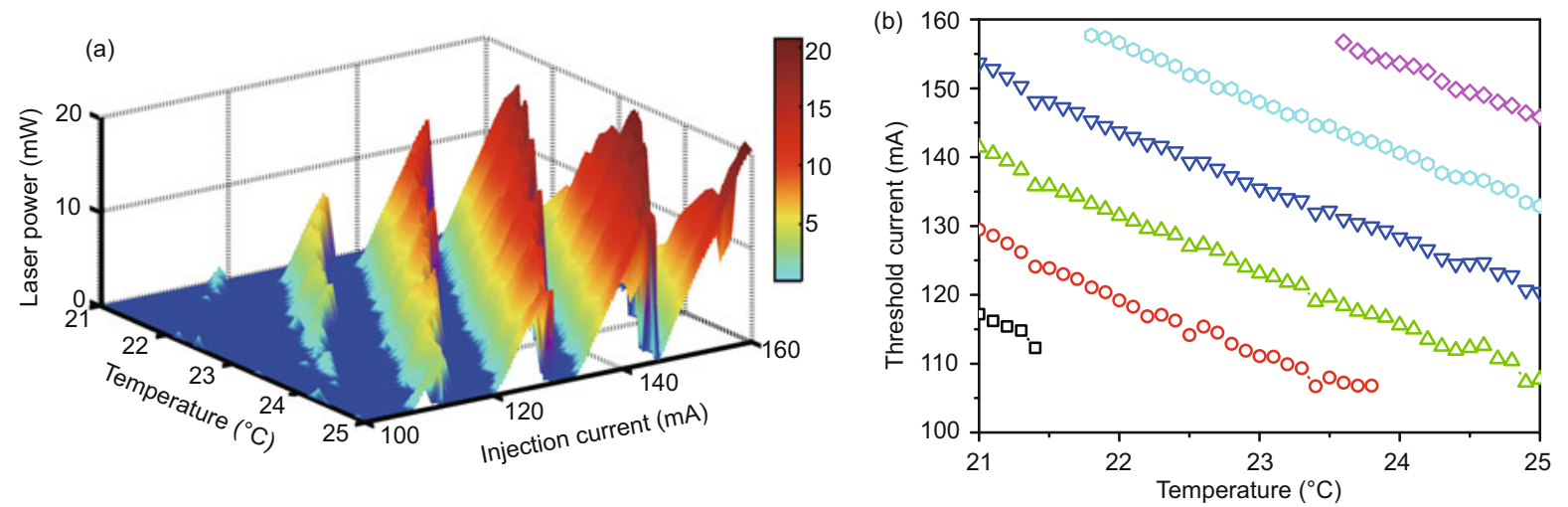

(c)
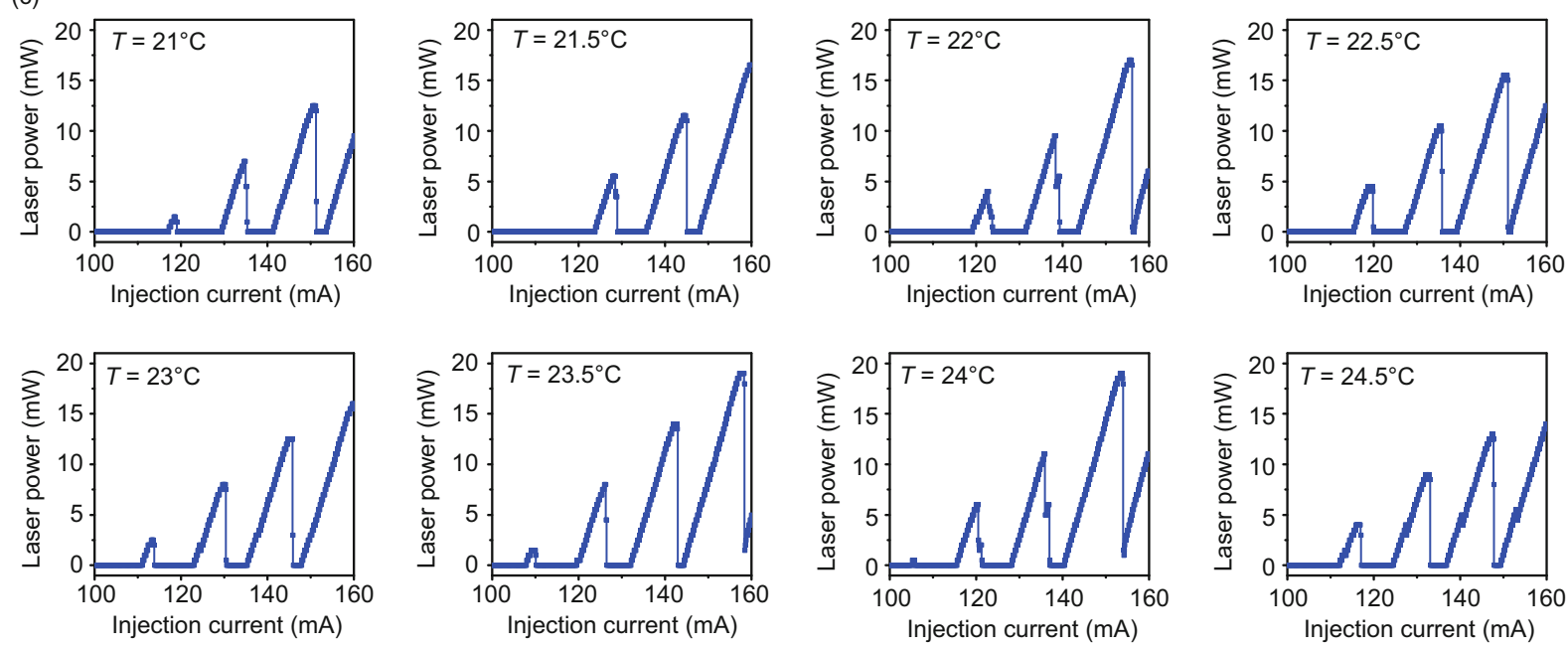

Figure 1 (a) The measured three-dimensional phase diagrams of multi-threshold laser power as a function of current and diode temperature. (b) Relation curves between threshold current and temperature of ECDL. Different data symbols represent relevant thresholds current of the multiple lasing regions showed in (a). A linear relationship can be observed, and the vertical displacements between adjacent lines are almost equal to $12.3 \mathrm{~mA}$. (c) Detailed power-current curves at different diode temperatures. Injection current is tuned with a step of $0.1 \mathrm{~mA}$ from 100 to $160 \mathrm{~mA}$ limited. There are more than one injection current thresholds which decrease while the temperature of ECDL is increased.

thresholds can exist at a certain operating diode temperature and that the laser revives at a new threshold after each "death". The multiple threshold currents as a function of the operating diode temperature are shown in Figure 1(b). A linear dependence of threshold current on diode temperature $T_{d}$ can be observed, and the vertical displacement between two adjacent lines are almost $12.3 \mathrm{~mA}$. The thresholds of the injection current $I$ decrease as the diode temperature $T_{d}$ is increased, which is in contrast to that in a usual ECDLs [28,29] where the threshold current increases with the diode temperature. In Figure 1(c), we show the detailed phase diagrams of our laser system (the measured power-current curves at different diode temperatures).

In our experiment, GaAs diode laser at a wave-length $\lambda=830 \mathrm{~nm}$ with the output facet coated with a multilayer antireflection coating was operated with the standard Litterman-Metcalf external cavity configuration. The size of the active region of laser diode is $0.1 \mu \mathrm{m} \times 2 \mu \mathrm{m} \times 300 \mu \mathrm{m}$ (height $\times$ width $\times$ length). The distance between laser diode and optical grating over a range from 35 to $1000 \mathrm{~mm}$ does not affect the results. The calculations shown in Figure 3(b) are based the laser parameters listed in Table 1, and the nonlinear gain effect has been included in calculation.

In the same diode laser, measurements during the last 38 months show exactly the same multi-threshold SOPT behavior. Once the laser is "alive", it operates at a single longitudinal mode, and the laser wavelength is identical at the beginnings of different thresholds. The beam profile of our ECDL is shown in Figure 2, which clearly shows that it operates at both single longitudinal mode and fundamental Gaussian traverse mode. When the laser is at "death" state, the measured fluorescence spectrum is as wide as $20 \mathrm{~nm}$. We have also measured the $P-I$ curves at different lasing wavelengths within the $20 \mathrm{~nm}$ gain range, and the multi-threshold SOPT consistently exists.

Figure 3(a) shows the dependence of the ECDL's output power as a function of the injection current $I$ at diode temperature $T_{d}=23^{\circ} \mathrm{C}$. The corresponding theoretical result obtained by soloving eq. (8) is given in Figure 3(b). The blue curves of both figures are the measured laser power as the injection current increased from 100 to $160 \mathrm{~mA}$, while the red curves are the measured results when the injection current is decreased. Four thresholds exist in both cases, with the first threshold appearing at $I=107 \mathrm{~mA}$ (there is no threshold below 

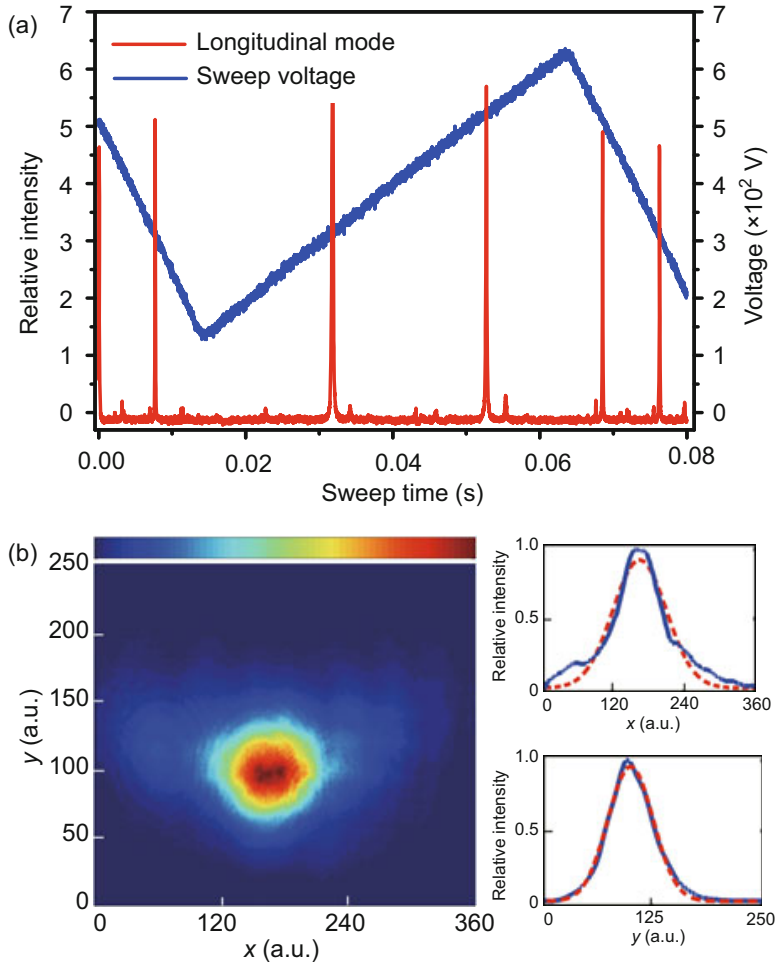

Figure 2 (a) The longitudinal mode of ECDL measured by scanning a high-fineness Fabry-Perot etalon. The blue line represents the sweeping voltage, and the red line denotes the longitudinal mode, which clearly shows that our ECDL is operating at single longitudinal mode. (b) The transverse mode profile of ECDL measured by a Laser Beam Profile Analyzer (LBP-2-USB) at half a meter away from the laser. The beam profile is shown in left picture, while the distributions of the relative intensity in $x$ direction and $y$ direction are shown in right figures (blue lines) with the Gaussian fitting curves (red lines). The measured waist of laser beam in $x$ direction is $879.93 \mu \mathrm{m}$ in the Gaussian fit, while the waist in $y$ direction is $478.61 \mu \mathrm{m}$ in the Gaussian fit.

$100 \mathrm{~mA}$ injection current). Each laser threshold is followed with a bi-stable state, which corresponds to the first-order phase transition [30]. In our case, when the injection current is gradually increased, the laser begins to lase at each threshold, and its power drops to zero almost suddenly after reaching a maximum value. However, if the injection current is decreased, the lasing action begins at a point that is lower than the previous maximum, and the output power decreases linearly to zero at the same threshold.

\section{Results and discussion}

Although Landau's theory of the SOPT [5, 6] and GinzburgLandau equations of superconductivity $[10,31]$ have been used to describe laser action [15-20,32], all fascinating SOPT explained so far in numerous physical systems such as spontaneous magnetization [21], superfluidity [22, 23] and superconductivity [24-26], are solely limited to single critical point category. We believe that the SOPT with multiple critical points described by eq. (2) is more general and even richer phenomenon than the conventional SOPT. The single threshold behavior is only a special case of the multi-
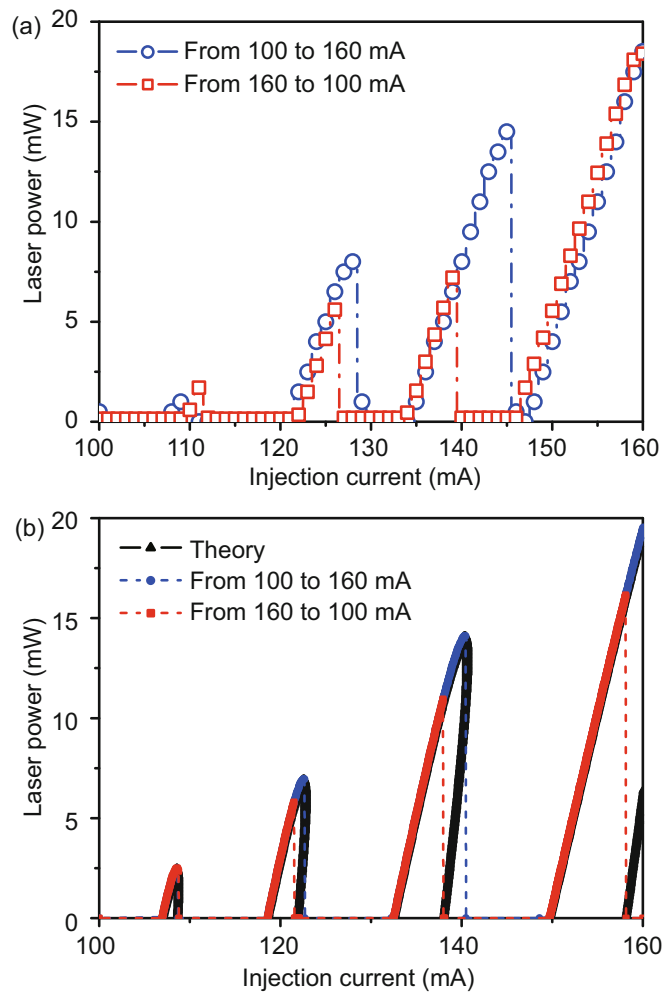

Figure 3 (a) Experimentally measured power-current dependence at diode temperature $23^{\circ} \mathrm{C}$. Blue circles were recorded when injection current was increased from 100 to $160 \mathrm{~mA}$ while red squares correspond to a decreasing injection current from 160 to $100 \mathrm{~mA}$. Hysteresis can be clearly observed. (b) Calculated results; blue and red lines are deduced from the calculated curve (black) which simulates the experimental results in (a). Theory could well explain the multi-threshold phenomenon and the optical bi-stability effect (Hysteresis) by comparing (a) and (b).

threshold SOPT as eq. (2) can be reduced to eq. (1) if $\phi(T)$ does not depend on the temperature $T$. The simple laser rate equations with the gain and cavity loss term $A-C$ depending linearly on population inversion only describes singlethreshold SOPT [15-20,33] or multi-threshld of first-order phase transition [20,33]. We have also observed the multithreshold SOPT phenomenon in other diode lasers operating at a wavelength of $852 \mathrm{~nm}$. The key is to carefully select the parameters to satisfy the gain and loss condition shown in eq. (4).

In summary, we proposed and demonstrated that once the effective temperature periodically affects the order parameter, a physical system described by Landau's theory of SOPT can be extended to exhibit multi-threshold SOPT. Our findings lead to a natural question: can multi-threshold SOPT also emerge in other physical systems, such as superconductivity, superfluidity, ferromagnetic system, Bose-Einstein condensates etc., which usually display single SOPT as in a laser?

The authors thank Wang Yiqiu and Xu Zhichao for helpful discussions. This work was supported by the National Natural Science Foundation of China (10874009 and 11074011). 
1 Zeng J, Li L, Liao Q, et al. Simulation of phase transition process using lattice Boltzmann method. Chinese Sci Bull, 2009, 54: 4596-4603

2 Ma J, Wu Y, Ying H, et al. Channel noise-induced phase transition of spiral wave in networks of Hodgkin-Huxley neurons. Chinese Sci Bull, 2011, 56: 151-157

3 Ding Y, Zhang G. Folding of a single polymer chain and phase transition. Chinese Sci Bull, 2009, 54: 1908-1911

4 Ai Q, Wang Y, Long G, et al. Two mode photon bunching effect as witness of quantum criticality in circuit QED. Sci China Ser G-Phys Mech Astron, 2009, 52: 1898-1905

5 Landau L D. Theory of phase transformations. Phys Z Sowjetunion, 1937, 11: 26-47, 545-555

6 Landau L D, Lifshitz E M. Statistical Physics. Oxford: Pergamon, 1958

7 Landau L D. The theory of superfluidity of helium II. J Exp Theor Phys USSR, 1941, 5: 71-90

8 Landau L D. On the hydrodynamics of helium II. J Exp Theor Phys USSR, 1944, 8: 1

9 Landau L D. On the theory of superfluidity of helium II. J Exp Theor Phys USSR, 1947, 11: 91-93

10 Ginzburg V L, Landau L D. On the theory of superconductivity. J Exp Theor Phys USSR, 1950, 20: 1064-1082

11 Onsager L. Crystal statistics I. A two-dimensional model with an orderdisorder transition. Phys Rev, 1944, 65: 117-149

12 Kaufman B, Onsager L. Crystal statistics III. Short-range order in a binary Ising lattice. Phys Rev, 1949, 76: 1244-1252

13 Pitaevskii L, Stringari S. Bose-Einstein Condensation. Oxford: Oxford University Press, 2003

14 Cummings F W, Johnston J R. Theory of superfluidity. Phys Rev, 1966, 151: 105-112

15 Graham R, Haken H. Laserlight - First example of a second order phase transition far from thermal equilibrium. Z Physik, 1970, 237: 31-46

16 DeGiorgio V, Scully M O. Analogy between the laser threshold region and a second-order phase transition. Phys Rev A, 1970, 2: 11701177

Open Access This article is distributed under the terms of the Creative Commons Attribution License which permits any use, distribution, and reproduction in any medium, provided the original author(s) and source are credited.
17 Haken H. Cooperative phenomena in systems far from thermal equilibrium and in nonphysical systems. Rev Mod Phys, 1975, 47: 67-121

18 Corti M, DeGiorgio V. Analogy between the laser and second-order phase transition: Measurement of "coexistence curve" and "susceptibility" for a single-mode laser near threshold. Phys Rev Lett, 1976, 36: 1173-1176

19 Haken H. Synergetics. New York: Springer-Verlag, 1977

20 Scully M O, Zubairy S. Quantum Optics. Cambridge: Cambridge University Press, 1997

21 Ising E. Beitrag zur theorie der ferromagnetismus. Z Phys, 1925, 31: 253-258

22 Kapitza P. Viscosity of liquid Helium below the $\lambda$ point. Nature, 1938, 141: 74-75

23 Tisza L. Transport phenomena in helium II. Nature, 1938, 141: 913-913

24 Kamerlingh-Onnes H. The superconductivity of mercury. Comm Phys Lab Univ Leiden, 1911, 122: 13-15 and 124: 21-25

25 Cooper L N. Bound electron pairs in a degenerate Fermi gas. Phys Rev, 1956, 104: 1189-1190

26 Bardeen J, Cooper L N, Schrieffer J R. Theory of superconductivity. Phys Rev, 1957, 108: 1175-1204

27 Ramsey N F. Thermodynamics and statistics mechanics at negative absolute temperature. Phys Rev, 1956, 103: 20-28

28 Chow W W, Koch S W, Sargent III M. Semiconductor-Laser Physics. New York: Springer-Verlag, 1994

29 Coldren L, Corzine S. Diode Lasers and Photonic Integrated Circuits. New York: Wiley, 1995

30 Scott J F, Sargent III M, Cantrell C D. Laser-phase transition analogy: Application to first-order transitions. Opt Commun, 1975, 15: 13-15

31 Rosenstein B, Li D. Ginzburg-Landau theory of type II superconductors in magnetic field. Rev Mod Phys, 2010, 82: 109-168

32 Staliunas K. Laser Ginzburg-Landau equation and laser hydrodynamics. Phys Rev A, 1993, 48: 1573-1581

33 Fang-Yen C, Yu C C, Ha S, et al. Observation of multiple thresholds in the many-atom cavity QED microlaser. Phys Rev A, 2006, 73: 041802 